\title{
BMJ Open A novel ACT-based video game to support mental health through embedded learning: a mixed-methods feasibility study protocol
}

Darren J Edwards (D) , ${ }^{1}$ Andrew $\mathrm{H}$ Kemp ${ }^{2}$

To cite: Edwards DJ, Kemp AH. A novel ACT-based video game to support mental health through embedded learning: a mixed-methods feasibility study protocol. BMJ Open 2020;10:e041667. doi:10.1136/ bmjopen-2020-041667

- Prepublication history and additional material for this paper is available online. To view these files, please visit the journal online (http://dx.doi.org/10. 1136/bmjopen-2020-041667)

Received 15 June 2020 Revised 06 0ctober 2020 Accepted 30 0ctober 2020

Check for updates

(C) Author(s) (or their employer(s)) 2020. Re-use permitted under CC BY-NC. No commercial re-use. See rights and permissions. Published by BMJ.

${ }^{1}$ Department of Public Health, Policy, and Social Sciences, Swansea University, Swansea UK

${ }^{2}$ Department of Psychology, Swansea University, Swansea, UK

Correspondence to Dr Darren J Edwards; D.J.Edwards@swansea.ac.uk

\section{ABSTRACT}

Introduction In recent years, serious video games have been used to promote emotional regulation in individuals with mental health issues. Although these therapeutic strategies are innovative, they are limited with respect to scope of treatment, often focusing on specific cognitive skills, to help remediate a specific mental health disorder. Objective Here, we propose a protocol for assessing the feasibility of a novel acceptance and commitment therapy (ACT)-based video game for young adults.

Methods and analysis The Medical Research Council (MRC) framework will be used for developing a complex intervention to design and test the feasibility of an ACTbased video game intervention using a mixed-methods approach involving qualitative and quantitative data. The primary outcomes will include feasibility testing of recruitment processes and the acceptability of the intervention through qualitative interviews, attendance and rates of attrition. Secondary outcomes will involve a series of quantitative questionnaires to obtain effect sizes for power analysis, allowing for the ideal sample size for an appropriately powered, randomised controlled trial to be determined.

Ethics and dissemination This study has been approved by the Psychology Department Research Ethics Committee (2020-4929-3923) at Swansea University in the UK. Dissemination activities will involve publications in peerreviewed journals, presentations at local and national conferences and promotion through social media. Trial registration number NCT04566042.

\section{INTRODUCTION}

Mental health issues such as anxiety and depression are a global problem of increasing concern, imposing considerable burden on society. The Global Burden of Disease project $^{1}$ has identified mental health disorders as a leading cause of disability globally, and suggest that there are 266 million cases of anxiety, and 253 million cases of major depressive disorder globally.

Unfortunately, the demand for mental health services far exceeds the available human resources able to meet this need in high-income and low-income
Strengths and limitations of this study

Mixed-methods approach to build a rich dataset on which conclusions will be drawn.

- Protocol follows established Medical Research Council (MRC) guidelines.

- In line with MRC guidelines and stage of game development, randomisation is not a component of this study.

- Aims are to assess feasibility, an important step in the development of complex interventions, although limiting conclusions able to be drawn.

andmiddle-income countries. This includes ever-growing treatment gaps ${ }^{2}$ and lags. ${ }^{3}$ These alarming increases have prompted the 2018 Lancet commission on global mental health to suggest that universal health coverage should include efforts to ensure the sustainable development of mental health. ${ }^{4}$ Innovations to promote accessibility to mental health treatments include technology such as telephone, internet and smartphone devices, augmenting the psychotherapeutic toolkit. ${ }^{5}$

Innovations in video gaming for remediating mental health issues have wide potential application. In the USA, over 164 million adults play video games, and at least three-quarters of all American families have at least one person who video games regularly. ${ }^{6}$ In the EU, $54 \%$ of the population play video games between the ages of 6 and 64 , where the average age of video gamers is 31 , and with a distribution of $46 \%$ female and $54 \%$ male. Of these, $77 \%$ play at least 1 hour per week, $16 \%$ play 1 hour per month, while only $7 \%$ play 1 hour per year. ${ }^{7}$ Given that such a large proportion of the Western population play video games, developing mental health training in the form of psychoeducation may have great potential for building psychological resilience and 
helping to better manage depression, anxiety and other forms of distress.

Technological developments for tackling such challenges include the exploitation of gamification. ${ }^{8}$ This involves the application of behavioural principles for controlling and modifying human behaviour, in which game design elements are used to increase human interaction with or without technology ${ }^{9}$ Some examples of gamification include gamifying the development of cognitive skills and emotional regulation by rewarding the completion of relevant tasks within complex video games ${ }^{10-13}$ in order to promote mental health.

When mental health-related video games are designed well, they have been shown to elevate self-esteem, selfefficacy, knowledge and awareness of illness, adherence to treatment and problem-solving skills, while lowering aggression. ${ }^{14}$ One of the most successful in the facilitation of mental health improvement is a serious video game-a complex game with multiple levels and settings—called PlayMancer (PM), which targets emotional regulation and was specifically designed to help manage impulse control disorders. ${ }^{11}{ }^{13}$ The objective of the PM game is to develop emotional and cognitive skills, while reducing impulsivity. The game has been shown to help treat bulimia nervosa by improving emotional regulation. ${ }^{15} 16$

PM also uses biofeedback (heart rate and heart rate variability) to model physiological and emotional reactions, feeding this information back to the participant. Some research has shown that facilitating awareness of one's own physiology (such as brain activity or cardiac function) enhances the treatment effects of mental health disorders (such as anxiety disorder, depression, obsessivecompulsive disorder (OCD) and schizophrenia) via selfregulation. ${ }^{17}$ Biofeedback has also been shown to improve impulse control difficulties, and attentional difficulties in bulimia nervosa and attention deficit hyperactivity disorder, ${ }^{151618}$ as well as symptoms of stress, anxiety and anger. ${ }^{19}$ The focus on physiological data in the psychotherapeutic context is gaining traction ${ }^{20-22}$ and has strong theoretical underpinnings. ${ }^{28-25}$

Within PM, there are three mini-games: 'The face of Cronos'; 'Treasures of the sea' and 'Sign of the Magupta'. Each of these mini-games were designed to train different skills, for example, 'The face of Cronos' and 'Treasures of the sea' develops planning skills, impulse control, coping skills, stress management and emotional self-regulation, while 'Sign of the Magupta' was designed to train relaxation, breathing techniques and improve physiological and emotional awareness. However, in the study ${ }^{15}$ PM was combined with sessions of cognitive behavioural therapy (CBT) and without a control measure (eg, CBT only) so the game was developed as an adjunct to traditional mental health training, and there is no real way of knowing the direct benefits of the game as opposed to training in CBT. In another study-a case study of a single participant playing PM-anxiety and impulsivity decreased prior to CBT. ${ }^{16}$ However, as this study was based on a single case, further studies using a randomised controlled trial (RCT) approach are needed to support and provide confidence to these findings.

Another game, Dojo, ${ }^{26}$ develops emotional regulation in adolescents with anxiety. It uses biofeedback (heart rate variability) and trains breathing techniques, muscle relaxation, positive thinking and guided imagery to attempt to reduce anxiety in adolescence. It also uses instructional videos and then engages players through immersive and emotionally evocative puzzles that challenge players to use newly acquired emotion regulation skills. However, a pre-post RCT with 1347 participants, compared with a standard 'off the shelf' commercial game 'Rayman 2' (whereby Rayman 2 was the control), reported no difference between Dojo and the control condition at reducing anxiety. As both of these games significantly reduced anxiety, it is possible that the reduction in anxiety was due distraction from anxiety-provoking thoughts, rather than developing psychoeducational skills per se. The authors concluded that crucial design issues need to be carefully thought through, which include a clear theoretical and therapeutic foundation. This includes appropriate methodology that can assess the causes of improvement, before developing and testing a serious video game for the treatment of mental health issues such as anxiety.

Commercial games (such as Rayman 2) have been explored in their unmodified forms for their effectiveness in helping with social skills training for autism, and cognitive distraction for anxiety and nausea for patients undergoing chemotherapy, ${ }^{27}$ with limited success. Evidence of generalisability of these games beyond gameplaying is limited, ${ }^{28}$ and this may be because they act as simple distractions rather than therapeutic psychoeducation applicable to participant's everyday lives. Another issue with many of these studies is that they often lack appropriate and rigorous methodology such as longitudinal follow-up, ${ }^{29}$ and a mixed-methodological approach that can assess the feasibility and acceptability of such interventions.

Given these issues, it is important to emphasise that the underlying theoretical basis for PM and Dojo relates to the development of emotional regulation skills. While emotional regulation has transdiagnostic application ${ }^{30}$ (ie, an intervention designed to treat multiple mental health conditions), these applications are not underpinned by theoretical frameworks that relate to formal psychotherapeutic interventions. Our proposed game is designed to be a comprehensive transdiagnostic intervention that will integrate a third wave behavioural therapy—as opposed to an adjunct to-acceptance and commitment therapy (ACT) ${ }^{31} 32$ It will therefore be a comprehensive strategy for managing many common mental health issues such as depression and anxiety and focus on developing clear psychoeducational skills in the form of psychological flexibility, well-being and resilience more generally. ${ }^{33}$

Given this comprehensive transdiagnostic focus on psychological flexibility through ACT - a fundamental component of general health and well-being ${ }^{34}$-our online video game may have much greater reach and 
impact than other serious video games such as PM, Dojo and many of the commercial games which are not based on third wave psychotherapy. Greater accessibility and impact have important implications for reducing treatment gaps and lags by making more mental health services available to those who need them.

One reason for choosing ACT in the game development process was pragmatism. For instance, researchers and clinicians may access freely available materials through the Association of Contextual Behavioural Science website (https://contextualscience.org/), and it does not require formal clinical training or accreditation to practice $^{35}$ which has important implications for translation to video game platforms. Another reason for choosing ACT as the basis for the game, is that it has a strong evidence base, and meta-analysis has found it to be efficacious for improving chronic pain, depression, psychotic symptoms, mixed anxiety, OCD, drug abuse and stress at work. ${ }^{36}$ This means it is an ideal general purpose therapeutic tool as opposed to restricted focus on for example impulsivity control such as the PM application ${ }^{11}{ }^{13}$ or simple relaxation skills for adolescence with anxiety, as is the focus of the Dojo game. ${ }^{26}$

ACT principles are designed to undermine the trappings of language in the form of difficult thoughts and associated feelings, and promotion of psychological flexibility. ${ }^{37}$ Language trappings can get individuals entangled and can prevent them from engaging with what is truly meaningful to them. The development of psychological flexibility through ACT is important because it is considered to be a fundamental component of well-being. ${ }^{34}$

The six ACT processes are: (1) the act of being in the here and now, present and mindful ${ }^{32}{ }^{38}$; (2) acceptance, the act of being aware and open to painful thoughts; (3) cognitive fusion, the act of recognising that thoughts are just thoughts and not to buy into them (the process of cognitive defusion $)^{39}$; (4) identifying values, values act as a life compass and direct us towards a life filled with purpose; (5) commitment to values orientation, which is the act of continually working towards a values orientation, even when an individual goes off track; (6) self as context (also called the transcendental self), is flexible and transcendent form of self. This involves the awareness of thoughts and feelings but the complete detachment from the literal meaning of thoughts. ${ }^{34}$

ACT has been usefully applied to many forms of mental health issues and has been applied in many different forms of delivery. This includes web-based interventions, ${ }^{40-42}$ teleconference ${ }^{43}$ and a downloadable app for smartphones. ${ }^{45}$ So, given the fact that video games can have positive well-being benefits, ${ }^{46}{ }^{47}$ and are applicable for therapeutic purposes, ${ }^{28}{ }^{48}$ a transdiagnostic ACT serious video-game may have great potential for similar reasons.

As ACT is a comprehensive transdiagnostic model and formal third wave cognitive behavioural approach, then its reach and impact in the form of a video game may be greater than that of PM or Dojo which were focused on simpler emotional skills development and biofeedback. For these reasons, we are proposing an ACT-based video game called 'ACTing Mind' as an innovative and accessible intervention to help individuals who struggle with anxiety, depression, stress and other forms of distress.

\section{Aims}

The research goals of this proposal are to determine the feasibility and acceptability of a novel ACT-based video game intervention for individuals with mental distress, in line with methodology described in the Medical Research Council (MRC) framework. ${ }^{49}{ }^{50}$ This proposal lays the foundation for which a pilot and full-scale RCT will be conducted to determine clinical effectiveness, and ultimately the recommendations of the importance of such innovations in primary care mental health policies and practices.

\section{METHODOLOGY}

This protocol has been developed following the Template for Intervention Description and Replication of Studies ${ }^{51}$ (see online supplemental appendix 1), as well as the MRC guidelines for the development of complex interventions. ${ }^{49}$ This includes five stages of development for a complex intervention including: (1) preclinical, involving a theoretical review of the literature (provided here), justifying the need for such an intervention for the proposed population; (2) phase I, modelling, involving the use of evidence to determine the components for underlying mechanisms. For this, we propose a qualitative element involving thematic analysis to enable us to understand what would be most beneficial to a general population with anxiety and depression; (3) phase II, conducting an exploratory pilot study (outlined here) to determine the feasibility of the methodology and design where some initial data can be collected; (4) phase III, an RCT to test the efficacy of the proposed intervention (in subsequent work); (5) phase IV, long-term follow-up to assess replicability.

\section{Public and patient involvement}

Key stakeholders were consulted and involved in the development of this protocol. The Patient Experience and Evaluation in Research (Patient Experience and Evaluation in Research (PEER): https://www.swansea. ac.uk/humanandhealthsciences/research-at-the-collegeof-human-and-health/patientexperienceandevaluationin researchpeergroup/) group in the College of Human and Health Sciences at Swansea University were consulted. This group represented members of the public, students and staff members, several of whom reported that they had experienced depression, anxiety or stress at some point in their lives and emphasised the need for innovative approaches of the delivery of mental health support. The feasibility design was explained to them, and they gave positive feedback about the nature of the design, intervention and outcome measures. 


\section{Study design}

This is a mixed-methods study which is designed to determine the feasibility and acceptability of an ACT-based video game for individuals with anxiety, depression and stress, and to increase psychological flexibility.

\section{Study setting}

The study will be conducted entirely online including the game and assessment (via the Qualtrics platform), and qualitative interviews (via the Zoom platform). Thus, potential participants will be able to access this study without restrictions, an important consideration for ongoing local lockdowns associated with the COVID-19 pandemic. Strict recommendations will require participants to ensure they are in a quiet room and without disruption for the duration of the study.

\section{Recruitment and consent}

We will recruit participants $(n=36)$ using purposive sampling, focusing on-unlike an opportunity samplethe types of participants needed for a full-scale RCT (ie, individuals with depression, anxiety and stress). The sample size is justified on the basis of past research reporting the median numbers of participants recruited for similar types of feasibility studies, ${ }^{52}$ incorporating both quantitative and qualitative elements.

\section{Eligibility criteria}

Participants will be recruited through general public mental health forums, social media and student populations. Thirty-six participants will take part in the study and they will be aged 18 years or older, be experiencing ongoing depression, anxiety and stress, and be able to read, write and speak English.

\section{Intervention}

This ACT-based video game intervention called 'ACTing Mind', developed and designed solely by DE, will involve students and members of the public attending five 1 hour sessions of an ACT-based video game. Each session will involve a different chapter of the video game, and each chapter will explore a different key component of ACT, with there being six in total (see table 1 for the different chapters and sessions involved).

These various components and principles of $\mathrm{ACT}^{31} 32$ will be taught within the different chapters of the game and through embedded learning. For example, the player will gain ACT skills while completing objectives within the game and without directly being taught these skills, but rewarded indirectly through points and progress awards. For instance, in one scene (see table 1) the character is confronted by painful memories, and the player has two choices: (1) to destroy the painful memories or (2) to accept these memories. If the player chooses to destroy the memories (avoidant-based strategies), the world becomes distorted and barriers form making the chapter impossible to complete. Alternatively, if the player chooses acceptance-based strategies they will be able to continue the game (hence in this scene they learn that acceptance is functionally better than avoidance).

The game will start with a depressed individual who has recently lost his wife in an accident, and is feeling depressed, isolated and lonely (see figure 1 as an example of this scene). Each chapter will reward ACT consistent behaviour with points on a 'psychoflexameter'. This is a dial on the border of the screen which indicates increased psychological flexibility as the player completes ACTbased tasks such as acceptance (chapter 1), being present (chapter 2), values and commitment (chapter 3), defusion (chapter 4) and self as context (chapter 5). ACT uses metaphors to help clients visualise the key processes of ACT. In the game, these metaphors are real representations, such as the 'sinking sand' game, 'dropping the rope' game, the 'chessboard game', the 'unwanted monster' game, the 'leaves on a stream' game (see table 1).

Within the game, the character will have to enter his own mind through a 'mind escape machine' (see figure 2 of this as an example of the character in his own mind). At the start of the game, it is explained through a brief historical story that he develops this machine to destroy and supress his unwanted painful thoughts and memories about his wife and loss. Once in his mind, he will learn that destroying or supressing thoughts creates barriers in his mind which prevents him from continuing the game. So, learning acceptance is crucial throughout this game and the character is rewarded for this through points and progress awards. Also, within the game, psychoeducation components explain thoughts as trappings of language which can often get people stuck in life, and prevent them from value consistent living, as well as the various emotional regulation strategies such as avoidance and acceptance.

As part of the study, in addition to playing the video game, participants will be asked to record events on a weekly basis, aspects of application of the ACT principles learnt in an everyday life in a journal. It is anticipated that greater adherence to the intervention in everyday life, and engagement with the journal will lead to greater success of the intervention (greater psychological flexibility).

\section{Data collection and management}

MSc students will have the opportunity to be involved in this study and will collect and process the data under supervision by project leads, DJE and AK. Questionnaires will be completed online through Qualtrics which will store raw data copies, and also be held on an encrypted university server. Names and other personally identifiable information will not be stored, and consent form information will not be associated with the raw or processed data, instead each participant will be given a unique identifier code. Similarity recorded interviewer transcripts will use identifier codes as opposed to personal information (eg, names). The project leads (DJE, AHK) will frequently audit all processes in data collection and processing to ensure that the procedures stated in this protocol are adhered to. 
Table 1 Overview of the 'ACTing Mind' intervention and everyday journal instructions

Session 1 (week

1): acceptance and openness to pain
Session 2 (week 1): being present (mindfulness)
- Chapter 1-Acceptance

- Introducing participants to the video game and ACT in everyday journal.

- A brief overview of the purpose of the programme and the content of each session.

- Explaining basic ACT tenets through introduction text of journal.

- Explaining the nature of painful thoughts and memories and getting caught up in the struggle explained through journal.

- Basic story context about the character being depressed and why, at start of video game.

- Explaining the objective of the video game, that is, to transcend form psychological inflexibility to psychological flexibility.

- Exercise, within the game there are choice, either to suppress, and break thoughts, or to accept and be open to them.

- Acceptance and openness are rewarded by psychological flexibility points on the 'psychoflexameter' and game progression, while suppression actions (breaking or suppressing painful memories) are punished with physical barriers, and sinking sand, which prevent the player from progressing in the game.

- A monster pulls against the player to prevent progress, but if the player fights with the monster, they get even more stuck (analogous to the drop the rope and sinking sand metaphor). Again, acceptance is important and must be learnt here.

- Reflecting in the journal about how this might be applied in life, and when this has occurred throughout the week daily.

- Chapter 2-Being present (mindfulness)

- Some instructions form the journal about being present and mindful is given, why it is useful and how to go about achieving with breathing exercises.

- The character is approached by monsters in the game in the past and future making him worry excessively about imaginary dangers, and reminding him of painful events.

- The game (in the form of the character's wife's ghost) instructs the player to be present, to focus on your breathing for $10 \mathrm{~min}$.

- As the participant learns and completes relevant psychological flexibility tasks psychological flexibility on the 'psychoflexameter' will increase, which rewards the player for being present.

- Reflecting in the journal about how this might be applied in life, and when this has occurred throughout the week daily.

Session 3 (week 2): values identification and commitment

- Chapter 3-Values identification and commitment

- Instructions about what are values (a life compass) explained through the journal.

- Acceptance and commitment to values orientation as opposed to avoidance behaviour is rewarded.

- There are challenges to reach goals which are linked to the character's values, such as scary weather and monsters.

- Psychological flexibility on the 'psychoflexameter' and game progress, will increase with values consistent behaviour which rewards the player for committing to values.

- Reflecting in the journal about how this might be applied in life, and when this has occurred throughout the week daily.

Session 4 (week 2): defusion

- Chapter 4-Defusion

- Instructions about what is cognitive fusion and defusion (holding self-stories lightly) explained through journal.

- The character goes back into the 'Mind Escape' machine but this time there is a flowing river with leaves (analogous to leaves on a stream metaphor).

- Some of the character's painful memories will beg the player to help them, but if the player interacts, barriers and quicksand appear, punishing the player and preventing them from progressing in the game (analogous to the sinking sand metaphor).

- The ghost of the character's wife eventually instructs the player to put the memories and thoughts onto the leaves and watch them flow down the river, without interacting with them, and to simply observe.

- Psychological flexibility on the 'psychoflexameter', will increase when all of the memories and thoughts as left to go down the stream, hence the player is rewarded for defusing.

- Reflecting in the journal about how this might be applied in life, and when this has occurred throughout the week daily. 
Table 1 Continued

Session 5 (week 3): self as context

- Chapter 5-Self as context

- Instructions about what is self as context (being the observer of your thoughts and not your thought) are explained through journal.

- The world starts to fall apart and becomes abstract, like a chess board.

- The player realises that they are the white pieces on the chessboard (analogous to chess board metaphor).

- The player is compelled by the game to beat the black pieces in the chess game.

- But the more the players fights against the black pieces, the more they lose points on the 'psychoflexameter' and cannot progress in the game.

- The player must let the battle play out, once they do, they become aware that they are the chess board (they become it) and realise they do not need to be part of the never-ending battle between the opposing forces.

- Finally, a bus arrives, memories of the character's wife beg the player to stay, and the monsters pull on player.

- The player needs to get onto the bus with the monsters to move towards their values, a new beginning (analogues to bus metaphor).

- Finally, the player has a choice, go back and change the events that led to your wife's death, or stay on the bus with the monsters.

- Trying to change events leads to a loss in points and prevents game progression.

- Only staying on the bus, towards values, and accepting the monsters allows the player to complete the game successfully.

- Reflecting in the journal about how this might be applied in life, and when this has occurred throughout the week daily.

\section{Outcome measures}

Questionnaires will be collected at three points in time (baseline, immediate postintervention and 3-month follow-up). Interview data will be collected immediate postintervention only.

\section{Demographic data}

Demographic measures will include age, sex, medication use, which will all be recorded through Qualtrics and assessed by DJE and AHK.

\section{Primary outcome measure}

The primary outcomes for feasibility are determined using MRC framework measure for developing a complex intervention. ${ }^{4950}$ As this is a feasibility study, the primary outcomes (in accordance with the MRC framework) will include the acceptability of the ACT-based video game intervention, the feasibility of the recruitment, outcome measures and intervention adherence.

\section{Acceptability}

- Number of people dropping out.

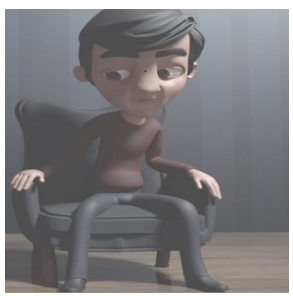

Figure 1 First scene in 'ACTing Mind', the character, Steve, is depressed and alone.
- Barriers for adoption of intervention as assessed through interviews.

- Number of sessions attended.

- Time dedicated to home journal.

- ACT principles adherence in everyday life setting (as recorded in journal and expressed through interviews).

- Experience, identifying whether participants had positive experience with the intervention and whether they wanted to continue to be part of the intervention.

Feasibility

- Number of participants who are willing to take part.

- Time taken to complete questionnaires.

- Number of complete and incomplete questionnaires.

\section{Secondary outcome measures}

Warwick-Edinburgh Mental Well-Being Scale ${ }^{53}$ : a measure of mental well-being with a focus on positive aspects of mental health. This measure has good internal

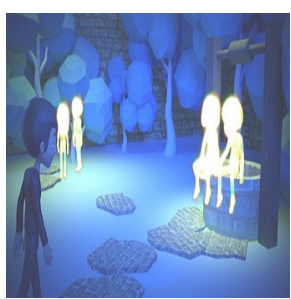

Figure 2 An example scene, where the character 'Steve' is in his own mind, and can see his own memories, through his Mindscape machine. 
consistency with a Cronbach's alpha coefficient of 0.89 (student sample) and 0.91 (general population sample).

Depression Anxiety Stress Scales: a short version of this measure and a measure of general psychological distress with good construct validity (confirmatory factor analysis of 0.94). It has good internal reliability as measured through Cronbach's alpha coefficients, which are 0.88 for depression, 0.82 for anxiety, 0.90 for stress and 0.93 for the total scale. ${ }^{54}$

Social connectedness (adapted from Russell's (1996) UCLA Loneliness Scale ${ }^{55}$ : this measure involves two questions: (1) "During social interactions, I feel 'in tune' with the person/s around me", and (2) "During social interactions, I feel close to the person/s". The Cronbach's alpha coefficients for these two items ranged from 0.80 to 0.98 $(\mathrm{M}=0.94, \mathrm{SD}=0.03) .{ }^{55}$

EuroQol five dimensions (EQ5D): the EQ5D is a measure for health-related quality of life. There are five components within this measure which assess mobility, self-care, usual activities, pain, discomfort and anxiety. It also has a visual analogue scale (VAS) for measuring current health status. Scores for these will be calculated for each of these five subsections as well as including the VAS and total EQ5D score of all five subsections. The EQD5 correlates well with other health-related questionnaires such as the 36-Item Short Form Survey ( $\mathrm{r}=0.61$, $\mathrm{p}<0.0001)$ and Parkinson's Disease Questionnaire (PDQ)-39 ( $\mathrm{r}=-0.75, \mathrm{p}<0.0001) .{ }^{56}$

Acceptance and Action Questionnaire-second version: this is a seven-item scale developed by Bond et a $\tilde{l}^{57}$ to measure psychological inflexibility, which involves the ability to accept and be open to difficult thoughts and feelings as well as to engage in valued behaviour in the presence of the difficult thoughts and feelings. A higher score indicates higher psychological inflexibility. The measure has good construct validity with a Cronbach's alpha coefficient of $0.84 .^{57}$

\section{Adherence to the intervention measure and trial}

Adherence will be measured in a variety of ways such as intervention feedback, treatment adherence through attrition rates as well as meta-data of relating to game log-in and log-out, as well as how long the game was played for and what sessions of the game were completed for each participant. Similar information can be recorded in Qualtrics for ensuring questionnaires are completed carefully. This includes length of times completing the questionnaire, and paying attention to reverse-scored questions.

\section{Sample size and statistical analysis}

Sample size recruited will help us determine whether it is possible to recruit sufficient numbers of participants to manage a full-scale RCT at a later date.

Quantitative data analysis: analysis will focus on descriptive statistics and feasibility outcomes of the questionnaires. While clinical effectiveness will not be formally evaluated at this stage, effect sizes will be explored for early evidence that the intervention shows promising signs (including ACT-related process measures). It is predicted that outcomes will improve, and any improvement will be identified using a one-way analysis of covariance with a single within-subjects factor (time). The effect sizes will also allow for a power calculation to be made which will allow for an approximation for a sample size required in a future trial (if indicated).

Qualitative data analysis: transcripts of focus group interview data will be generated from digital audiorecordings of in-depth, face-to-face semi-structured interviews (all online and via a password-protected room in Zoom). In-depth semi-structured interviews will form the core topics to be discussed (see table 2), while leaving space and scope for the identification and exploration of unforeseen information that may emerge. Insights from this will allow for further development and improvement of the intervention, along with the quantitative data in line with the MRC guidelines. ${ }^{49} 50$

Thematic analysis will then be conducted which will explore key overarching themes that may emerge from the focus group interviews following standardised guidelines. ${ }^{58}$ The interview questions are based on other novel ACT-based protocols. ${ }^{40} 59$ The data will be analysed after the study has been completed. We will follow the inductive and deductive code development as outlined by Fereday and Muir-Cochrane ${ }^{60}$ to ensure necessary rigour. Any key overarching themes identified which relate to feasibility of the study design of the acceptability of the intervention, as well as potential adverse effects, will be explored and reported.

The focus groups will comprise 4 to 6 groups with 6 to 10 individuals in each group as has been suggested as optimal in other studies. ${ }^{61}$ The interview will take place at the end of the intervention (week 3). It will explore various aspects of the intervention such as perceived process of change, barriers to intervention adherence, trial process and any adverse effects, which help supplement the quantitative approach. Process of change questions indicate whether the participant learnt anything about ACT, and felt any positive change in their life due to participating in the intervention. The question relating to barriers explores any problems and difficulties they had with the intervention. Another question will be asked to elicit suggestions for improvement relating to game or study design. Acceptability questions and process of change in one's life relate to whether the participant accepted the intervention and used skills they learnt through the intervention in daily life. The question relating to the trial process will determine whether there were any difficulties or limitations of the trial itself such as whether the instructions were clear and how it could be improved. Finally, the question on adverse effects explores whether there were any potential unforeseen negative consequences of the intervention.

\section{Limitations of the study}

This study protocol has limitations. First, while physiological measures would ideally be collected to measure 
Table 2 Qualitative interview protocol for the focus groups

\begin{tabular}{|c|c|}
\hline Acceptability and feasibility & $\begin{array}{l}\text { How would you describe your experience of taking part in 'ACTing Mind' video game } \\
\text { programme? }\end{array}$ \\
\hline Accessibility of intervention & $\begin{array}{l}\text { If this intervention were rolled out as a video game app, do you think you would download it? } \\
\text { Would you appreciate the accessibility? }\end{array}$ \\
\hline Acceptability & $\begin{array}{l}\text { What was the aspect of the programme that you liked the most? What was your favourite activity } \\
\text { within the game (or applied to your everyday life)? }\end{array}$ \\
\hline Barriers & Were there any difficulties to taking part? \\
\hline $\begin{array}{l}\text { Implementing change in } \\
\text { everday life }\end{array}$ & $\begin{array}{l}\text { Do you practice mindfulness, acceptance, defusion and values? How often? Could you apply } \\
\text { what you have learnt through video game intervention to the real world in everyday events? Will } \\
\text { you apply this new knowledge to everyday events? }\end{array}$ \\
\hline Processes of the trial & $\begin{array}{l}\text { Was there anything you liked, or disliked about the study? How could we improve this study? } \\
\text { Were all the instructions clear? }\end{array}$ \\
\hline Adverse effects & $\begin{array}{l}\text { Did you feel that any aspect of the intervention may have made worse any aspect of your } \\
\text { anxiety, depression or stress? Were there any adverse effects that you can recognise due to the } \\
\text { intervention? }\end{array}$ \\
\hline
\end{tabular}

variables such as heart rate variability, the COVID-19 pandemic limits our capacity to do this. However, the present study will provide important data on which such measures could be collected, analysed and interpreted in a future trial. Second, it could be questioned why there is no control condition in this study. Our response to this potential criticism is that the aim of the present study is to assess feasibility and-in line with the MRC guidelines ${ }^{49}{ }^{50}$ - has not been designed to be a full-scale RCT given the current research phase. Once the feasibility component is completed, a control condition will be introduced, which allow for the intervention condition to be compared with control, and as part of a full trial. Finally, although we would like to have ability to monitor the participant more directly, to ensure adherence to the intervention, we are sensitive to privacy issues associated with, for example, capturing participants' identity from the computers video camera. To mitigate this limitation, we have opted for less invasive procedures for measuring intervention adherence that will include logging metadata of the game such as log in and out times, as well as completion of game sections. Several questions in the questionnaire are also reverse scored to ensure participants are paying attention.

\section{Protocol amendments}

If the protocol is amended in any way, it will be communicated to relevant parties immediately, such as to participants, journal and ethics committee.

\section{Ethics and dissemination}

This study has received ethical approval from Swansea University Psychology Department ethics committee (2020-4920-3923). Participants will be informed of their rights to confidentiality and to leave the study at any time and without penalty. Both qualitative and quantitative data will be held on a password-protected computer accessible only to researchers DJE and AHK. The data will be anonymised with a unique identifier code, and any personally identifiable information will be removed.

Dissemination will involve peer-reviewed journals; leading national and international conferences, social media and public events and through general public health engagement such as talks at schools, the Welsh Government and engagement with annual science festivals including 'a pint of science'.

\section{Impact of intervention}

The potential impact of this study is far reaching as it will add to the growing set of online resources which support psychological resilience, flexibility and well-being. These resources are designed to be easy to access and are ideal for situations where travel is limited due to physical (disability) or situational (coronavirus) immobility. Such interventions can help alleviate widely reported mental health treatment gaps ${ }^{2}$ and lags, ${ }^{3}$ associated with the widely reported scarce human resources needed to provide mental health support for the many individuals who need it. The 2018 Lancet commission on global mental health argued that sustainable development 
of mental health should be an essential component of universal health coverage. ${ }^{4}$ Technological innovation of mental health support services, in the form of video games, may be one means to achieve this sustainability and a reduction in the treatment gap and lag.

\section{Ancillary and poststudy care}

Postintervention care has not been anticipated given this is a low-level (low-risk) intervention. Of course, all participants will be given a debrief form which will signpost individuals to the relevant free well-being services such as the Samaritans.

Acknowledgements The authors would like to thank Professor Louise McHugh (University College Dublin), who provided some very helpful feedback about the gamification of an ACT-based approach through the utilisation of behavioural principles.

Contributors DJE developed the intervention. DJE and AHK agreed on a set of outcomes. DJE wrote the first draft of the protocol and DJE and AHK then revised the subsequent drafts of the protocol. Both authors helped to revise the manuscript for intellectual content and agreed on the final version prior to submission for peer review.

Funding The authors have not declared a specific grant for this research from any funding agency in the public, commercial or not-for-profit sectors.

Competing interests At the time of writing this, DJE is discussing with Agor IP at Swansea University the potential to commercialise the described video game as a mobile application; however, at this time no agreements have been made or signed. AHK has no competing interests.

Patient consent for publication Not required.

Provenance and peer review Not commissioned; externally peer reviewed.

Supplemental material This content has been supplied by the author(s). It has not been vetted by BMJ Publishing Group Limited (BMJ) and may not have been peer-reviewed. Any opinions or recommendations discussed are solely those of the author(s) and are not endorsed by BMJ. BMJ disclaims all liability and responsibility arising from any reliance placed on the content. Where the content includes any translated material, BMJ does not warrant the accuracy and reliability of the translations (including but not limited to local regulations, clinical guidelines, terminology, drug names and drug dosages), and is not responsible for any error and/or omissions arising from translation and adaptation or otherwise.

Open access This is an open access article distributed in accordance with the Creative Commons Attribution Non Commercial (CC BY-NC 4.0) license, which permits others to distribute, remix, adapt, build upon this work non-commercially, and license their derivative works on different terms, provided the original work is properly cited, appropriate credit is given, any changes made indicated, and the use is non-commercial. See: http://creativecommons.org/licenses/by-nc/4.0/.

ORCID iD

Darren J Edwards http://orcid.org/0000-0002-2143-1198

\section{REFERENCES}

1 Whiteford H, Ferrari A, Degenhardt L. Global burden of disease studies: implications for mental and substance use disorders. Health Aff 2016;35:1114-20.

2 Patel V, Maj M, Flisher AJ, et al. Reducing the treatment gap for mental disorders: a WPA survey. World Psychiatry 2010;9:169-76.

3 Wang PS, Berglund PA, Olfson M, et al. Delays in initial treatment contact after first onset of a mental disorder. Health Serv Res 2004;39:393-416.

4 Patel V, Saxena S, Lund C, et al. The Lancet commission on global mental health and sustainable development. The Lancet 2018;392:1553-98.

5 Kazdin AE, Blase SL. Rebooting psychotherapy research and practice to reduce the burden of mental illness. Perspect Psychol Sci 2011;6:21-37.

6 ESA. 2019 essential facts about the computer and video game industry, 2020. Available: https://www.theesa.com/esa-research/ 2019-essential-facts-about-the-computer-and-video-game-industry/
7 ISFE. ISFE key facts 2019, 2020. Available: https://www.isfe.eu/isfekey-facts/

8 Linehan C, Kirman B, Roche B. Gamification as behavioral psychology. In the gameful world: approaches, issues, applications. Cambridge, Massachusetts: MIT Press, 2015.

9 Deterding S, Dixon D, Khaled R, et al. From game design elements to gamefulness: defining "gamification". Proceedings of the 15th international academic MindTrek conference: Envisioning future media environments, 2011:9-15.

10 Jon Hobbs L, Yan Z. Cracking the walnut: using a computer game to impact cognition, emotion, and behavior of highly aggressive fifth grade students. Comput Human Behav 2008;24:421-38.

11 Jiménez-Murcia S, Fernández-Aranda F, Kalapanidas E, et al. Playmancer project: a serious videogame as an additional therapy tool for eating and impulse control disorders. Stud Health Technol Inform 2009;144:163-6.

12 Ducharme P, Wharff E, Hutchinson E, et al. Videogame assisted emotional regulation training: an act with RAGE-Control case illustration. Clin Soc Work J 2012;40:75-84.

13 Fernández-Aranda F, Jiménez-Murcia S, Santamaría JJ, et al. Video games as a complementary therapy tool in mental disorders: PlayMancer, a European multicentre study. J Ment Health 2012;21:364-74.

14 Santamaria JJ, Antonio S, Fernando F-A, et al. Serious games as additional psychological support: a review of the literature. $J$ Cyber Ther Reh 2011;4:469-76.

15 Fagundo AB, Santamaría JJ, Forcano L, et al. Video game therapy for emotional regulation and impulsivity control in a series of treated cases with Bulimia nervosa. Eur Eat Disord Rev 2013;21:493-9.

16 Giner-Bartolomé C, Fagundo AB, Sánchez I, et al. Can an intervention based on a serious videogame prior to cognitive behavioral therapy be helpful in Bulimia nervosa? a clinical case study. Front Psychol 2015;6:982.

17 Schoenberg PLA, David AS. Biofeedback for psychiatric disorders: a systematic review. Appl Psychophysiol Biofeedback 2014;39:109-35.

18 Howard R, Schellhorn K, Lumsden J. A biofeedback intervention to control impulsiveness in a severely personality disordered forensic patient. Personal Ment Health 2013;7:168-73.

19 Pawlow LA, O'Neil PM, Malcolm RJ. Night eating syndrome: effects of brief relaxation training on stress, mood, hunger, and eating patterns. Int J Obes Relat Metab Disord 2003;27:970-8.

20 Dana DA. The Polyvagal theory in therapy: engaging the rhythm of regulation (Norton series on interpersonal neurobiology). New York: WW Norton \& Company, 2018.

21 Lehrer PM. Heart rate variability biofeedback and other psychophysiological procedures as important elements in psychotherapy. Int J Psychophysiol 2018;131:89-95.

22 Tulip C, Fisher Z, Bankhead $\mathrm{H}$, et al. Building wellbeing in people with chronic conditions: a qualitative evaluation of an 8-week positive psychotherapy intervention for people living with an acquired brain injury. Front Psychol 2020;11:66.

23 Kemp AH, Koenig J, Thayer JF. From psychological moments to mortality: a multidisciplinary synthesis on heart rate variability spanning the continuum of time. Neurosci Biobehav Rev 2017;83:547-67.

24 Mead J, Wilkie L, Gibbs K, et al. Rethinking wellbeing: toward a more ethical science of wellbeing that considers current and future generations, 2019. Available: https://www.authorea.com/doi/full/10. 22541/au.156649190.08734276

25 Kemp A. Fisher, social ties, health and wellbeing: a literature review and model, in neuroscience and social science. Berlin: Springer, 2017: 397-427.

26 Scholten H, Malmberg M, Lobel A, et al. A randomized controlled trial to test the effectiveness of an immersive 3D video game for anxiety prevention among adolescents. PLoS One 2016;11:e0147763.

27 Colder Carras M, Van Rooij AJ, Spruijt-Metz D, et al. Commercial video games as therapy: a new research agenda to unlock the potential of a global pastime. Front Psychiatry 2017;8:300.

28 Griffiths MD, Kuss DJ, de Gortari ABO. Videogames as therapy: an updated selective review of the medical and psychological literature. JPHIM 2017;5:71-96.

29 Zayeni D, Raynaud J-P, Revet A. Therapeutic and preventive use of video games in child and adolescent psychiatry: a systematic review. Front Psychiatry 2020;11:36.

30 Sloan E, Hall K, Moulding R, et al. Emotion regulation as a transdiagnostic treatment construct across anxiety, depression, substance, eating and borderline personality disorders: a systematic review. Clin Psychol Rev 2017;57:141-63.

31 Hayes S. Acceptance and commitment therapy. Washington, DC: American Psychological Association, 2009. 
32 Hayes Steven C, Kirk D. Acceptance and commitment therapy: the process and practice of mindful change. New York: Guilford Press, 2011.

33 Dindo L, Van Liew JR, Arch JJ. Acceptance and commitment therapy: a transdiagnostic behavioral intervention for mental health and medical conditions. Neurotherapeutics 2017;14:546-53.

34 Kashdan TB, Rottenberg J. Psychological flexibility as a fundamental aspect of health. Clin Psychol Rev 2010;30:865-78.

35 Harris R. Act made simple: an easy-to-read primer on acceptance and commitment therapy. Oakland, California: New Harbinger Publications, 2009.

36 Öst L-G. The efficacy of acceptance and commitment therapy: an updated systematic review and meta-analysis. Behav Res Ther 2014;61:105-21.

37 Hayes SC, Strosahl KD, Bunting K, et al. What is acceptance and commitment therapy?, in a practical guide to acceptance and commitment therapy. Berlin: Springer, 2004: 3-29.

38 Strosahl K, Wilson K. Acceptance and commitment therapy: an experiential approach to behavior change. New York: Guilford Press, 1999.

39 Hayes SC. Get out of your mind and into your life: the new acceptance and commitment therapy. Oakland, California: New Harbinger Publications, 2005.

40 Edwards DJ, Rainey E, Boukouvala V, et al. Novel ACT-based eHealth psychoeducational intervention for students with mental distress: a study protocol for a mixed-methodology pilot trial. BMJ Open 2019;9:e029411.

41 Viskovich S, Pakenham KI. Pilot evaluation of a web-based acceptance and commitment therapy program to promote mental health skills in university students. J Clin Psychol 2018;74:2047-69.

42 Levin ME, Haeger JA, Pierce BG, et al. Web-based acceptance and commitment therapy for mental health problems in college students: a randomized controlled trial. Behav Modif 2017;41:141-62.

43 Herbert MS, Afari N, Liu L, et al. Telehealth versus in-person acceptance and commitment therapy for chronic pain: a randomized noninferiority trial. J Pain 2017;18:200-11.

44 Bricker JB, Mull KE, Kientz JA, et al. Randomized, controlled pilot trial of a smartphone APP for smoking cessation using acceptance and commitment therapy. Drug Alcohol Depend 2014;143: :87-94.

45 Levin ME, Haeger J, Pierce B, et al. Evaluating an adjunctive mobile APP to enhance psychological flexibility in acceptance and commitment therapy. Behav Modif 2017;41:846-67.

46 Johnson D, Jones C, Scholes L, et al. Videogames and wellbeing: a comprehensive review. young and well cooperative research centre, Melbourne, 2013. Available: https://www.youngandwellcrc.org.au/ wp-content/uploads/2014/03/Videogames_and_Wellbeing.pdf

47 Vella K, Johnson D, Hides L. Positively playful: when videogames lead to player wellbeing. Proceedings of the First International
Conference on Gameful Design, Research, and Applications, 2013:99-102.

48 Villani D, Carissoli C, Triberti S, et al. Videogames for emotion regulation: a systematic review. Games Health J 2018;7:85-99.

49 Craig P. Developing and evaluating complex interventions: following considerable development in the field since 2006, MRC and NIHR have jointly commissioned an update of this guidance to be published in 2019, 2019. Available: https://mrc.ukri.org/documents/ pdf/complex-interventions-guidance/

50 Craig P, Dieppe P, Macintyre S, et al. Developing and evaluating complex interventions: the new medical Research Council guidance. BMJ 2008;337:a1655.

51 Hoffmann TC, Glasziou PP, Boutron I, et al. Better reporting of interventions: template for intervention description and replication (TIDieR) checklist and guide. BMJ 2014;348:g1687.

52 Billingham SAM, Whitehead AL, Julious SA. An audit of sample sizes for pilot and feasibility trials being undertaken in the United Kingdom registered in the United Kingdom clinical research network database. BMC Med Res Methodol 2013;13:104.

53 Tennant R, Hiller L, Fishwick R, et al. The Warwick-Edinburgh mental well-being scale (WEMWBS): development and UK validation. Health Qual Life Outcomes 2007;5:63.

54 Henry JD, Crawford JR. The short-form version of the depression anxiety stress scales (DASS-21): construct validity and normative data in a large non-clinical sample. Br J Clin Psychol 2005:44:227-39.

55 Kok BE, Coffey KA, Cohn MA, et al. How positive emotions build physical health: perceived positive social connections account for the upward spiral between positive emotions and vagal tone. Psychol Sci 2013;24:1123-32.

56 Schrag A, Selai C, Jahanshahi M, et al. The EQ-5D--a generic quality of life measure-is a useful instrument to measure quality of life in patients with Parkinson's disease. J Neurol Neurosurg Psychiatry 2000;69:67-73.

57 Bond FW, Hayes SC, Baer RA, et al. Preliminary psychometric properties of the acceptance and action Questionnaire-II: a revised measure of psychological inflexibility and experiential avoidance. Behav Ther 2011;42:676-88.

58 Braun V, Clarke V. Using thematic analysis in psychology. Qual Res Psychol 2006;3:77-101.

59 Saracutu M, Edwards DJ, Davies $\mathrm{H}$, et al. Protocol for a feasibility and acceptability study using a brief ACT-based intervention for people from Southwest Wales who live with persistent pain. BMJ Open 2018;8:e021866.

60 Fereday J, Muir-Cochrane E. Demonstrating rigor using thematic analysis: a hybrid approach of inductive and deductive coding and theme development. Int J Qual Methods 2006;5:80-92.

61 Morgan DL. Focus groups. Annu Rev Sociol 1996;22:129-52. 